\title{
On a Model Mechanism for the Spatial Patterning of Teeth Primordia in the Alligator
}

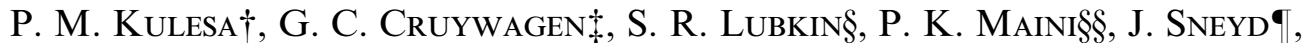 \\ M. W. J. Ferguson $\uparrow$ and J. D. Murray§
}

\begin{abstract}
$\dagger$ Division of Biology, Beckman Institute (139-74), California Institute of Technology, Pasadena, CA, 91125, U.S.A.; \$Sea Fisheries Research Institute, Rogge Bay, 8012 Capetown, South Africa; §Department of Applied Mathematics, University of Washington, Seattle, WA 98195-2420, U.S.A.; §§ Centre for Mathematical Biology, Mathematical Institute, 24-29 St Giles', Oxford OX13LB, U.K.; - Department of Mathematics, University of Canterbury, Christchurch, NZ; and $\uparrow$ School of Biological Sciences, The University of Manchester, 3.239 Stopford Building, Oxford Road, Manchester, M13 9PT, U.K.
\end{abstract}

(Received on 12 October 1994, Accepted in revised form on 14 December 1995)

\begin{abstract}
We propose a model mechanism for the initiation and spatial positioning of teeth primordia in the alligator, Alligator mississippiensis. Detailed embryological studies by Westergaard \& Ferguson (1986, 1987, 1990) show that jaw growth plays a crucial role in the developmental patterning of the tooth initiation process. Based on biological data we develop a reaction-diffusion mechanism, which crucially includes domain growth. The model can reproduce the spatial pattern development of the first seven teeth primordia in the lower half jaw of A. mississippiensis. The results for the precise spatio-temporal sequence compare well with detailed developmental experiments.
\end{abstract}

(C) 1996 Academic Press Limited

\section{Tooth Development: an Introduction}

\subsection{TOOTH DEVELOPMENT AS A MODEL SYSTEM}

The morphogenesis of teeth in the vertebrate jaw is a process which incorporates many aspects of development, from self-organization and spatial patterning to tissue interaction and cell differentiation. Thus, the formation of teeth represents a model system to study mechanisms which may generalize to other developmental processes. The first signs of developing teeth primordia are the formation of placodes: localized thickenings of the oral epithelium. Through a series of complex epithelialmesenchymal interactions, which occur while the jaw is growing, these clumps of epithelial cells give rise to the development of tooth germs in the jaw

$\dagger$ Author to whom correspondence should be addressed. mesenchyme. Subsequently, the placodes are either resorbed or differentiate to form functioning teeth. In the present paper we focus on the spatio-temporal development of pattern, in terms of the condensation of epithelium into teeth primordia, observed in the tooth initiation process.

\subsection{BENEFITS OF CROCODILIAN EMBRYOLOGY}

For palaeontologists, teeth represent a means of measuring evolutionary change. Tooth shape, age and formation may be used to study adaptive aspects of evolution as well as any genetic links to dentition development within the animal kingdom. Many of the mechanisms involved in tooth development are common to the development of several systems. Therefore, mathematical models of dentition formation may generalize to other developmental systems. 
The study of early mammalian dentition development has been hindered by the inaccessibility of embryonic processes in vivo. This problem can be overcome by experimental investigation of dental embryology in the crocodilia, in particular Alligator mississippiensis. This animal exhibits the unique combination of developing in an external egg and possessing the most mammal-like snout and palate of all the crocodilia, having transcended some 230 million years of evolutionary time without undergoing major morphological alterations (Ferguson, 1981). An understanding of the basic developmental mechanisms involved in crocodilian embryology may provide clues to the morphogenesis of advanced structures found in higher vertebrates (Ferguson, 1981).

\section{Previous Theory and Experiments}

\subsection{TheORETICAL MODELS}

The early embryological investigations of dentition development in reptiles (Röse, 1894; Woerdeman, 1919; Osborn, 1971) form the basis for descriptive models of tooth formation, which in general fall into either a prepattern or dynamic model category. Prepattern models are characterized by substances called morphogens and rely on the concept of positional information (Wolpert, 1969). Diffusion of a morphogen through a tissue creates a gradient in concentration of the morphogen. In the tooth initiation process, prepattern models propose that a primordium is formed when cells respond to differences in the morphogen concentration: this is the essence of positional information. Edmund (1960) postulated what is called the Zahnreihe theory, that teeth were determined by initiation waves which activated various prepatterned tooth sites. Osborn (1971), however, showed that the Zahnreihe theory could not account for any known tooth initiation pattern in embryos, and was the first to propose a dynamic patterning model for tooth initiation (Osborn, 1978). Dynamic models describe the development of pattern as self-generating, a dynamic process which occurs as a result of growth of the system.

The clone model (Osborn, 1978) postulates that teeth are initiated from one or more clones of neural crest cells that form patterns as a result of the dynamic growth of the clone. In the model, a clone source has edges, called progress zones, which may expand in either direction, anterior or posterior along the jaw. Within the growing clone, a primordium is initiated when cell divisions give rise to competent tissue. This primordium generates a zone which inhibits the initiation of further local primordia (Osborn, 1971). As the clone expands, new primordia are initated when space and sufficient tissue become available. The size of the inhibitory zones and the growth of the clone determine the number of primordia. Osborn (1993) has extended the clone model to incorporate tooth shape formation.

\subsection{EXPERIMENTAL DATA}

A series of detailed investigations on the embryonic development of the dentition of the lower (Westergaard \& Ferguson, 1986, 1987) and upper (Westergaard \& Ferguson, 1990) jaws of $A$. mississippiensis has been completed from days 1 to 75 . Accurate sequences of initiation and replacement were derived and the development of individual teeth followed through the 65-day incubation period. The principal findings suggest that initiation of teeth is related to: (1) jaw growth; (2) the distance between existing teeth and; (3) the size and developmental maturity of the latter. The experimental results confirm the inadequacies of the Zahnreihe theory and have also led to a rejection (Westergaard \& Ferguson, 1986) of the clone model (Osborn, 1978), based on the criticism that new teeth do not develop in the sequence suggested by the growing clone. Osborn (1993) has suggested that the teeth, in a jaw quadrant of an alligator embryo, may develop from not one, but three clones. From the data of either the upper or lower jaw (Westergaard \& Ferguson, 1986, 1987, 1990), even in the case of three clones, it is not possible to match the experimental data with the model (Osborn, 1978). Clearly, any proposed model mechanism for tooth initiation must be capable of reproducing the known spatial and temporal sequence of teeth primordia in the alligator.

\section{Biological Basis of Tooth Formation}

\subsection{TOOTH INITIATION PROCESS}

Development of an individual tooth is characterized by a series of processes, culminating in formation of the mature shape and the mineralized tissues of dentine, enamel and cementum. The first indication of tooth formation is the appearance of a localized condensation of cells in the epithelium: a placode. Below the thickened epithelium, mesenchymal cells are attracted to this site and aggregate to form a localized condensation underneath the tooth site: the mesenchymal cell aggregations form the dental papillae. Early primordia degenerate into the mesenchyme and are resorbed or shed, while later 
primordia continue to develop into functioning teeth. Subsequent primordia form in a similar manner in a precise spatial and temporal sequence. In the present paper we concentrate on the primary early event of tooth morphogenesis, namely the initiation process for tooth placodes in the epithelium.

\subsection{SPATIAL PATTERNING OF THE TEETH PRIMORDIA}

In their work on the alligator dentition, Westergaard \& Ferguson $(1986,1987)$ observed a distinct pattern of tooth initiation in A. mississippiensis during development. The spatial sequence of the first seven primordia is shown in Fig. 1. The first tooth primordium, called the dental determinant, forms in the anterior part of the lower jaw, but is not the most anterior tooth to form. Tooth initiation spreads from the dental determinant both forwards and backwards in the jaw. Interstitial primordia form in the growing spaces between earlier primordia, closer to the more mature of the two, while new end primordia form at some distance from the former end primordia. This fact led Westergaard \& Ferguson $(1986,1987)$ to propose the existence of an inhibition zone around each developing tooth primordium (as previously suggested by Osborn, 1971), which diminishes with tooth maturity. A major conclusion from this experimental work is that teeth primordia formation is directly related to jaw growth and that the number of primordia increases exponentially. If we count the number of teeth which are formed as of each developmental day, we find an unmistakeable exponential relationship for the early development, and a Gompertz-like growth in the number of teeth primordia over the entire course of development. Figure 2 shows the temporal sequence of the first seven, while Fig. 3 shows the temporal sequence of the remaining primordia.

A mechanical model for initiation of the dental determinant has been presented by Sneyd et al. (1993). In their model, mechanical forces generated by the epithelial layer, mediated by a cellular adhesion
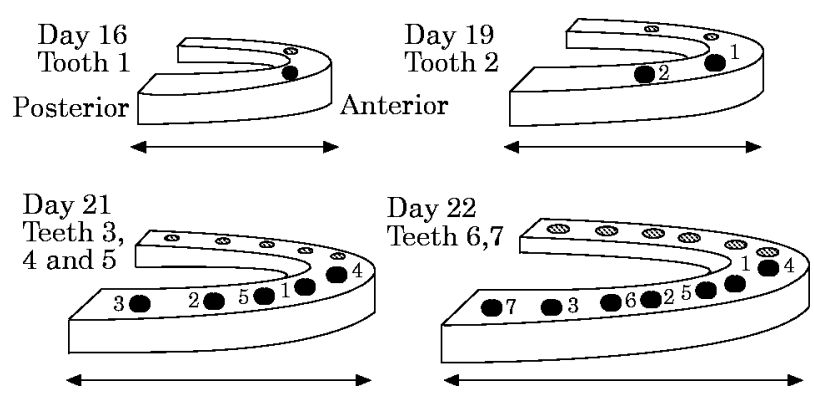

FIG. 1. Spatial pattern of the first seven teeth primordia in the lower right half jaw of Alligator mississippiensis (from Westergaard \& Ferguson, 1986).

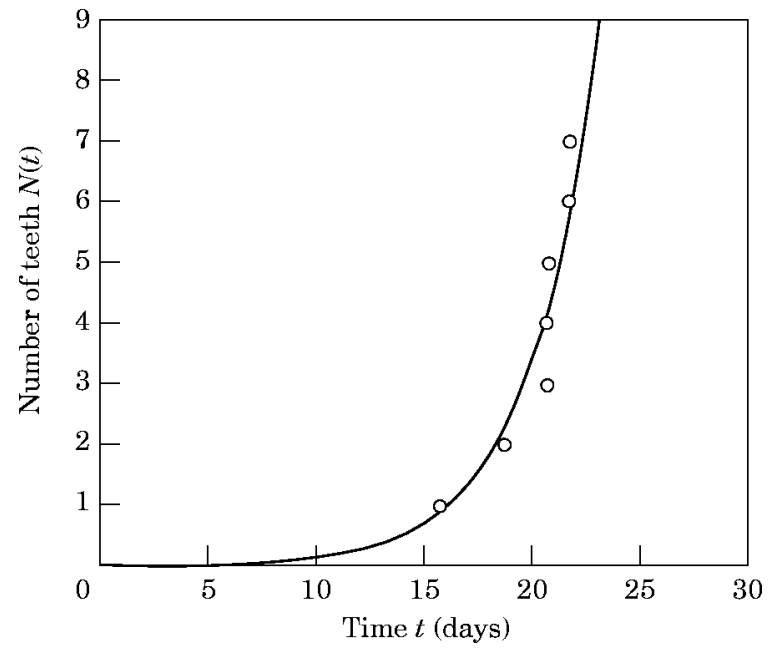

FIG. 2. Number of teeth $N(t)$ vs. time $t$ (days of incubation) for the first seven teeth primordia in the lower half jaw of Alligator mississippiensis. The solid line represents $N(t)=N_{0} \exp (r t)$; where $N_{0}=0.0066$ and $r=0.31 /$ day (derived from Westergaard \& Ferguson, 1986).

molecule (CAM), induce a local increase in cell density to form a placode. Their numerical calculations showed that the dental determinant would form in the proper spatial position only if CAM production in the region of the developing placode increased on a timescale much faster than that of jaw growth. The formation of subsequent teeth primordia was not investigated.

Here we propose a chemical mechanism in the epithelial layer which captures the tooth initiation and spatial patterning of the first seven teeth primordia in a simple system of reaction-diffusion equations. We

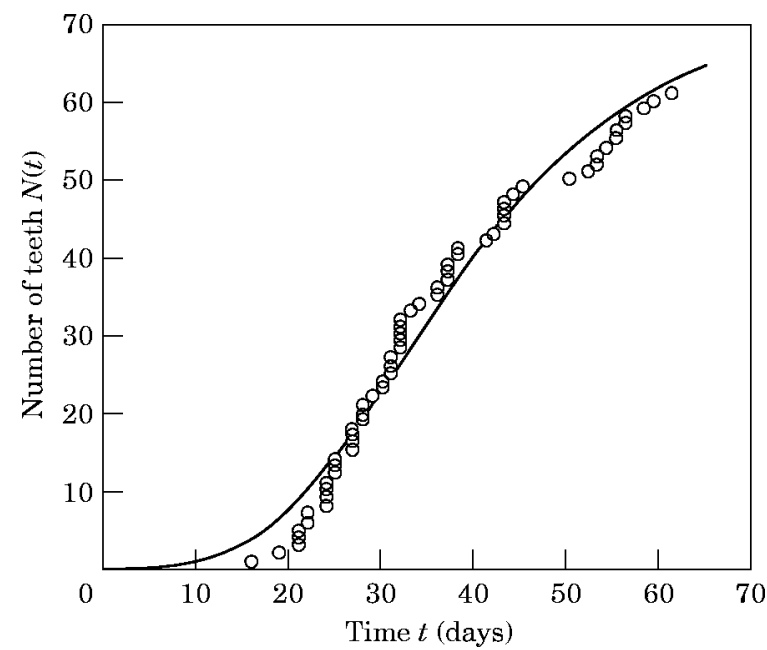

FIG. 3. Number of teeth $N(t)$ vs. time $t$ (days of incubation) in the lower half jaw of Alligator mississippiensis. The solid line represents a Gompertz curve: $N(t)=N_{1} \exp \left[-N_{2} \exp (-r t)\right.$; $N_{1}=71.8, N_{2}=8.96, r=0.0685 /$ day (derived from Westergaard \& Ferguson, 1986, 1987). 
consider only the first seven teeth, as the formation of subsequent primordia is complicated by resorbing older teeth, cell differentiation and differential jaw growth. In Section 3.3 we discuss the experimental work which motivates our use of a chemical mechanism. Besides the work on reptiles, there have been considerable experimental investigations of tooth morphogenesis in mammals (for example, mice). We consider the relevant experimental evidence on tooth initiation in mice as a guide for our modelling assumptions on alligator dentition.

\subsection{TOOTH INITIATION EXPERIMENTS IN MICE}

Tooth formation in mammals is complicated by the differences in tooth shape (that is, molar, canine, incisor): position in the jaw is closely related to tooth shape (Miles \& Grigson, 1990). Development of the mammalian dentition involves both regional and temporal patterning (MacKenzie et al., 1992). The source of dental patterning information had long been thought to reside in neural crest cells. Transplant experiments (Andreas, 1946, 1949; Wagner, 1949, 1959; Horstadius \& Sellman, 1946; Sellman, 1946; Noden, 1983) showed that neural crest cells (perhaps a specific subpopulation) contain developmental information to initiate tooth formation. However, extensive epithelial-mesenchymal recombination experiments (Mina \& Kollar, 1987; Lumsden, 1988; Kollar \& Mina, 1991) indicate that oral epithelium can stimulate tooth formation from any neural crestderived mesenchyme. These results have yet to be demonstrated in reptiles. However, it is not unreasonable to assume that tooth development in reptiles (particularly those with certain mammalian characteristics) is initiated by the epithelium.

Experimental investigations are presently focused on finding the molecular mechanisms involved in regulating the epithelium to stimulate the mesenchyme. In work on mice, Partanen \& Thesleff (1985) identified an epidermal growth factor (EGF) which caused proliferation of dental epithelium. Kronmiller et al. (1991) then demonstrated the necessity for the presence of this epidermal growth factor during tooth initiation by showing that initiation did not occur when epidermal growth factor was chemically blocked. This work was followed (Hill et al., 1989; Robert et al., 1989; Monaghan et al., 1991) by identification of the presence of certain homeobox genes in the vicinity of tooth initiation sites. In particular, MacKenzie et al. (1992) have shown that the Msx-2 gene is expressed in mouse oral epithelium in early dental placodes. This represents the earliest known marker for sites of dental initiation whose gene product may be important in causing dental initiation and patterning. In a similar study, MacKenzie et al. (1991) concluded that Msx-1 is expressed exclusively in the condensing mesenchymal cells of dental papillae and follicles from the onset of dental development, with no expression in the dental epithelia. Further investigations have revealed that Msx-1 and Msx-2 are expressed as a result of epithelial-mesenchymal interactions (Jowett et al., 1993) and that bone morphogenetic protein, BMP-4, released by the epithelium can induce Msx-1 in the underlying mesenchyme (Vainio et al., 1993).

\section{Model Mechanism}

Our aim is to show that the proposed class of mechanisms for the initiation and spatial positioning of the teeth primordia, which we now construct, are sufficient to explain the pattern of tooth sites in A. mississippiensis.

We base our model on the following biological data.

(i) We consider the lower half jaw in A. mississippiensis. Comparisons of tooth initiation sequences and positions between left and right sides of the jaw for both the same and other specimens of A. mississippiensis show no evidence of differences (Westergaard \& Ferguson, 1986, 1987). (ii) We model the jaw region where the teeth primordia form as a one-dimensional domain, namely the anterior-posterior axis. Experimental results (Westergaard \& Ferguson, 1986) show that tooth sites have very little lateral shift from an imaginary line drawn from anterior to posterior along the jaw line. (iii) Based on the evidence discussed in Section 3.3, we consider the spatial patterning of the primordia as an epithelial process. (iv) An integral part of the mechanism is the inclusion of an exponentially growing domain, that is a jaw growing at a constant strain rate. This assumption of exponential growth is based on the experimental results shown in Fig. 2.

\subsection{MODEL EQUATIONS}

The experimental evidence (Section 3.3) shows tooth initiation patterning to be an epithelially derived process which produces a pattern in the epithelial layer. This, together with the identification of the involvement of epidermal growth factor, bone morphogenetic protein and certain homeobox genes, suggests a chemical mechanism for initiation of a primordium, where certain substances react and diffuse so as to form gradients of concentration. These chemical gradients in turn stimulate an area of the epithelium to form a placode. Turing (1952) suggested that chemicals can react and diffuse to produce 
steady-state heterogeneous spatial patterns of chemical concentration. He showed that with the introduction of small spatial perturbations, under certain conditions a linearly stable uniform steady state of chemical concentrations could be driven unstable by diffusion to form spatially inhomogeneous patterns. Since this work, many reaction-diffusion systems which are capable of satisfying Turing's conditions for pattern formation have been studied [see Murray (1989) for an extensive review].

We propose a dynamic reaction-diffusion mechanism mediated by an inhibitor related to the concentration of EGF. We assume the existence of an inhibitory substance whose concentration decreases as the concentration of EGF increases, and vice versa. We first consider a fixed domain without growth, and denote position along the jaw (measured from the posterior end of the jaw) by $z$ and time by $t$. Let the concentrations of a substrate, activator and inhibitor be denoted respectively, by $u(z, t), v(z, t)$ and $c(z, t)$. We start with a jaw domain of length $L$, and non-dimensionalize all lengths with respect to $L$ : that is, the dimensionless space denoted by $\xi=z / L$ is such that $0 \leqslant \xi \leqslant 1$. We take, solely by way of example, the simple Schnakenberg (1979) system as our model reaction-diffusion system, which is identical to the system presented by Gierer \& Meinhardt (1972). We modify the system by assuming that the source of $u$ is controlled by $c$, which itself satisfies a reactiondiffusion equation. We first describe the model mechanism on a fixed domain and then consider the experimentally realistic situation of an exponentially growing jaw. The non-dimensionalized equations for the substrate, $u$, and activator, $v$, are, on a fixed domain $0 \leqslant \xi \leqslant 1$ :

$$
\frac{\partial u}{\partial t} \quad=\quad \gamma\left[h c-u+u^{2} v\right]+\frac{\partial^{2} u}{\partial \xi^{2}}
$$

$$
\begin{array}{ccc}
\begin{array}{c}
\text { Rate of change } \\
\text { in substrate } \\
\text { concentration }
\end{array} & =\begin{array}{c}
\text { Reaction kinetics } \\
\text { and bifurcation } \\
\text { 'parameter', } c
\end{array} & \text { Diffusion } \\
\frac{\partial v}{\partial t} & = & \gamma\left[b-u^{2} v\right]
\end{array} \quad+d \frac{\partial^{2} v}{\partial \xi^{2}}(2)
$$

where $d$ is the ratio of diffusion coefficients and $\gamma, b$ and $h$ are positive parameters. For a certain range of parameter values and domain size larger than some minimum, the reaction-diffusion system $(1,2)$ is capable of producing steady-state spatial patterns in $u$ and $v$. By varying one or more of the parameters in eqns (1) and (2), the system can select a stable heterogeneous state or spatial pattern. When the inhibitor, $c$, is above a threshold value, pattern formation in $u$ and $v$ is inhibited. For $c$ below this threshold, the pattern formation mechanism is switched on, via diffusion-driven instability, and spatial pattern forms in $u$ and $v$ when the subthreshold portion of the domain is large enough. The analysis for this is now quite standard [see, for example, Murray (1989)]. The equation for the inhibitor $c$, also on the fixed domain $0 \leqslant \xi \leqslant 1$ is taken to be

$$
\frac{\partial c}{\partial t} \quad=-\quad \delta c+p \frac{\partial^{2} c}{\partial \xi^{2}}
$$

\section{Rate of change \\ in inhibitor $=$ Degradation Diffusion}

where $p$ is the diffusion coefficient and $\delta$ is a first order degradation constant. We make the further assumptions that there is a source of $c$ at the posterior end of the jaw and to simulate the existence of an inhibition zone, each developing tooth primordium is a source of $c$.

The representation of the tooth primordium becoming a source of the inhibitor $c(\xi, t)$ is dictated by the biology since it has been observed experimentally that there is a local zone of inhibition around a newly formed primordium. We define a new tooth site where the substrate, $u(\xi, t)$, first crosses a threshold on a subdomain of $0 \leqslant \xi \leqslant 1$. This turns on a new tooth source $\bar{c}_{i}$ of $c$ at this site, which simulates a zone of inhibition. The concentration of this tooth source we model with logistic growth,

$$
\begin{aligned}
\frac{\mathrm{d}}{\mathrm{d} t} \bar{c}_{i}=k_{1} \bar{c}_{i}\left(1-\frac{\bar{c}_{i}}{k_{2}}\right) \text { at } \xi=\xi_{i} \text { for } t>t_{i} \\
(i=i \text {-th tooth })
\end{aligned}
$$

where $k_{1}, k_{2}>0, c\left(\xi_{i}, t\right)=\bar{c}_{i}(t)$ for all $t>t_{i}$, and $\bar{c}_{i}\left(t_{i}\right)=c\left(\xi_{i}, t_{i}\right)$.

\subsection{EXPONENTIAL JAW GROWTH AND THE EFFECT ON THE MODEL EQUATIONS}

Experimental evidence requires that the pattern arises dynamically as a result of jaw growth and not as the result of a prepattern of tooth initiation sites. This is a crucial element in the mechanism. Based on evidence of exponential jaw growth (Fig. 2), we assume that jaw length, $L=L(t)$, grows at a constant strain rate, $r$, according to

$$
\frac{\mathrm{d} L}{\mathrm{~d} t}=r L
$$


and so

$$
L(t)=L_{0} e^{r t}
$$

with $L_{0}$ constant.

The growing domain dilutes the chemical concentrations, but a larger domain produces replacement chemicals via the source terms in (1), (2) and (4). This results in an additional term, due to jaw growth, in each of the model equations (1-3) (see Appendix). We then transform the system on a growing domain to one on a fixed domain $0 \leqslant x \leqslant 1$ using the coordinate transformation $x=\xi e^{-r t}$. The non-dimensionalized equations become:

$$
\begin{array}{cccc}
\frac{\partial u}{\partial t} & =\gamma\left[h c-u+u^{2} v\right]-r u+\left(e^{-2 r t}\right) \frac{\partial^{2} u}{\partial x^{2}} \\
\begin{array}{c}
\text { Rate of } \\
\text { change in } \\
\text { substrate } \\
\text { concentration }
\end{array} & \begin{array}{c}
\text { Reaction kinetics } \\
\text { and bifurcation } \\
\text { 'parameter' } \\
c(x, t)
\end{array} & \begin{array}{c}
\text { Dilution } \\
\text { due to jaw } \\
\text { growth }
\end{array} & \text { Diffusion } \\
& \text { growth } &
\end{array}
$$

$$
\begin{array}{ccccc}
\frac{\partial v}{\partial t} & =\gamma\left[b-u^{2} v\right]-r v \quad+d\left(e^{-2 r t}\right) \frac{\partial^{2} v}{\partial x^{2}} \\
\begin{array}{c}
\text { Rate of } \\
\text { change in } \\
\text { activator } \\
\text { concentration }
\end{array} & = & \text { Reaction kinetics } & \begin{array}{c}
\text { Dilution } \\
\text { due to jaw } \\
\text { growth }
\end{array} & \text { Diffusion } \\
\frac{\partial c}{\partial t} & = & -\delta c & -r c \quad+p\left(e^{-2 r t}\right) \frac{\partial^{2} c}{\partial x^{2}} \\
\begin{array}{c}
\text { Rate of } \\
\text { change }
\end{array} & = & \text { Degradation } & \begin{array}{c}
\text { Dilution } \\
\text { due to jaw } \\
\text { growth }
\end{array} & \text { Diffusion }
\end{array}
$$

Each eqn (6-8) has a dilution term, due to jaw growth, and a new time dependent diffusion coefficient which arises from the coordinate transformation to the scaled domain in $x$. The boundary conditions are

$$
\begin{gathered}
u_{x}(0, t)=u_{x}(1, t)=v_{x}(0, t)=v_{x}(1, t)=0 \\
c_{x}(1, t)=0 \\
c(0, t)=c_{0}(t) \\
c(x, 0)=c_{0}(x)
\end{gathered}
$$

where $c(0, t)=c_{0}(t)$ is a decreasing function corresponding to a source term (Fig. 6). Also $c(x, 0)=c_{0}(x)$ is a monotonically decreasing function (with $c_{0}^{\prime}(1)=0$ ). The condition (9a) implies no flux or $u$ and $v$ at either end of the domain, while for $c$ there is no flux at the anterior end (9b).

\subsection{HOW THE MECHANISM WORKS}

We assume there is an initial source of inhibitor, $c$, at the posterior end of the jaw $(x=0)$. This chemical diffuses through the jaw epithelium, degrades, and is diluted by growth. As the jaw grows, $c$ decreases further towards the anterior end until it crosses below the critical threshold on a sufficiently large subdomain to drive the activator-inhibitor system unstable. When the subdomain on which $c$ is below the threshold, has grown large enough, a single mode spatial pattern in $u$ and $v$ will start to grow. Eventually, the activator concentration, $u$, crosses an upper threshold which triggers initiation of a placode (tooth primordium) fixing the spatial position of tooth 1 (Fig. 4).

Experimental evidence (Westergaard \& Ferguson, 1986) suggests that the dental determinant (and each subsequent tooth primordium) becomes a source of inhibitor. Thus, in our model, when $u$ grows above a certain threshold, we make the location of the peak in $u$ a source of $c$. Mathematically, this is equivalent to an internal boundary condition at each tooth

$$
c\left(x_{i}, t\right)=\bar{c}_{i}(t)
$$

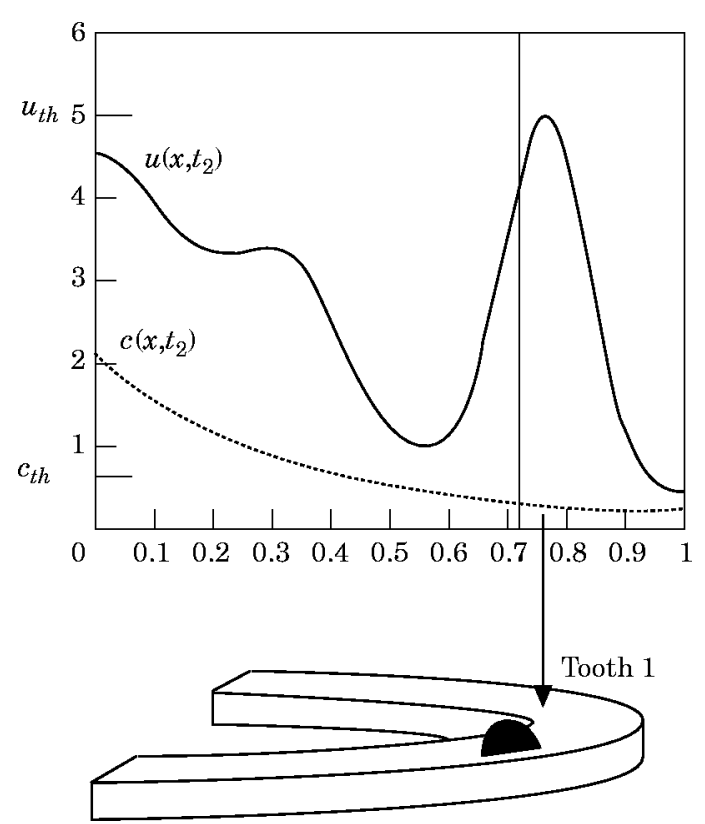

FIG. 4. The concentration profiles for $u$ and $c$ are shown for $t=t_{1}>0$. As the jaw grows, $c$ crosses below the threshold, $c_{t h}$. When the subdomain on which $c$ is below the threshold has grown large enough, a spatial pattern in $u$, starts to grow and eventually crosses a threshold, $u_{t h}$, which triggers initiation of a placode setting the spatial position of tooth 1 . The numerical simulations use a finite difference scheme (NAG D03-PCF) with the model parameters (the same for Figs 4-7):

$$
\gamma=40, \delta=0.2, h=1, \mathrm{~b}=2, p=0.5
$$

$r=0.01, d=150, k_{1}=0.3, k_{2}=1.0$. 


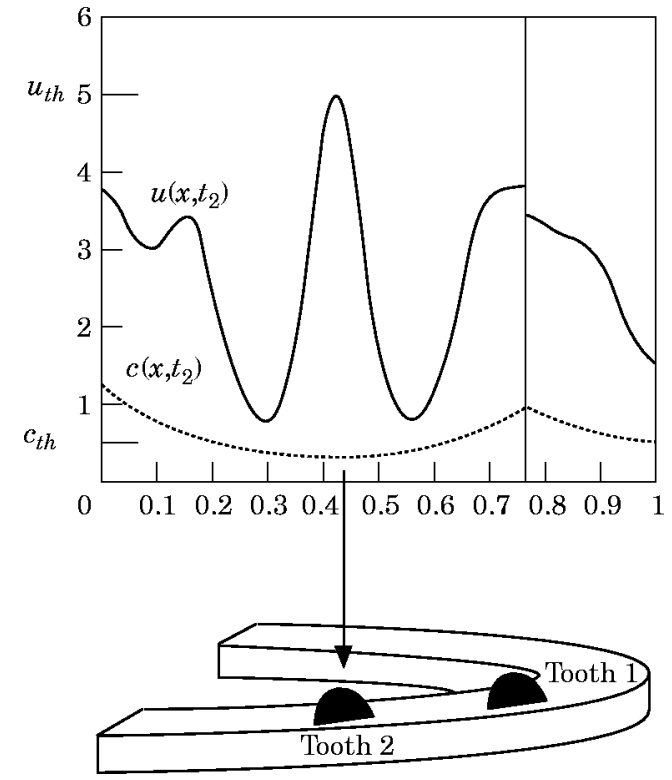

FIG. 5. As the jaw grows, $c$ drops below the threshold, $c_{t h}$, in the region between the two sources, the second primordium forms in the region where $u$ again crosses the patterning threshold, $u_{t h}$.

for $x=x_{i}$ and $t>t_{i}$, where $\bar{c}_{i}(t)$ is the solution of (4). There are now two sources. As the jaw grows, $c$ drops below the critical threshold in the region between the two sources and the next tooth position forms in the posterior end of the jaw. The second primordium forms in the region where $u$ again crosses the patterning threshold, and the tooth that is initiated becomes another source of $c$ (Fig. 5). In this manner, tooth development proceeds: $c(x, t)$ dips below a threshold, causing a local pattern to form when the domain size is sufficient; in forming the pattern, $u$ crosses a threshold, and creates a source of $c$, hence another tooth primordium. Subsequent primordia appear in a similar manner.

\section{Results}

The mathematical analysis of a reaction-diffusion system, such as the substrate-activator (1), (2) which are capable of generating pattern, typically begins with a linear analysis about a uniform steady state. This analysis determines certain parameter regimes where the steady state can be driven unstable by spatially heterogeneous perturbations. The steady state evolves to an inhomogeneous spatial pattern in $u$ and $v$ whose nature is governed by the parameters of the equations, the size of the domain and the boundary conditions.

The system (1), (2), with $c$ constant in space and time, has a uniform steady state which bifurcates to an inhomogeneous spatial pattern in $u$ and $v$. The spatial dependence of $c(x, t)$ in the reaction term of (1) requires that any steady state be inhomogeneous. This precludes using current techniques for further analysis without simplification of the equations. Spatially dependent reaction-diffusion systems are becoming important (Steinbock et al., 1995) and have been considered from a theoretical point of view (Auchmuty \& Nicolis, 1975; Mimura \& Nishiura, 1979). In a more applied format, Cantrell \& Cosner (1991) considered a reaction-diffusion equation with a piecewise continuous spatially dependent term in the reaction kinetics. Benson et al. (1993), also considered a piecewise continuous spatially dependent diffusion coefficient. However, in both analyses, there existed a homogeneous steady state about which the underlying equations could be linearized. Eigensolutions could then be calculated analytically on separate domains and then matched across the discontinuities.

Our model equations (1-3) are not amenable to such analytical methods because of the lack of a homogeneous steady state around which to linearize. However, we still expect the model to be driven unstable by heterogeneous perturbations, and to evolve from a heterogeneous state into another spatially heterogeneous state. The formation of pattern has two requirements here. For a pattern to form, $c$ must be below a threshold and the domain large enough to support a pattern. The reaction kinetics of the $u, v$ system are in effect waiting for the domain to grow and for $c$ to dip below the required threshold before they can produce a spatial pattern for the teeth primordia. The biology dictates that exponential growth be incorporated into these equations and the analysis of (6-9) becomes much more challenging. We therefore use numerical simulation techniques. The system of eqns (6-9) was solved numerically using a finite difference spatial discretisation and the method of lines to integrate in time (NAG-D03PCF). The procedure was first to solve for the position, $x_{1}$, of tooth 1 on $0 \leqslant x \leqslant 1$, then partition this interval into two subdomains and solve for the position, $x_{2}$, of tooth 2 . Biologically, we assume that the thickened epithelial cell condensations of a new tooth form a physical barrier to chemical diffusion of $u$ and $v$, and the new tooth becomes a source of inhibitor. This is mathematically equivalent to the internal boundary conditions

$$
\begin{aligned}
& u_{x}\left(x_{i}, t\right)=v_{x}\left(x_{i}, t\right)=0 \text { for } t>t_{i}, \\
& u\left(x_{i}, t_{i}\right)=u_{t h}, u_{x}\left(x_{i}, t_{i}\right)=0 .
\end{aligned}
$$

The numerical simulations for the first seven teeth followed in this way. The initial conditions for $u, v$ 


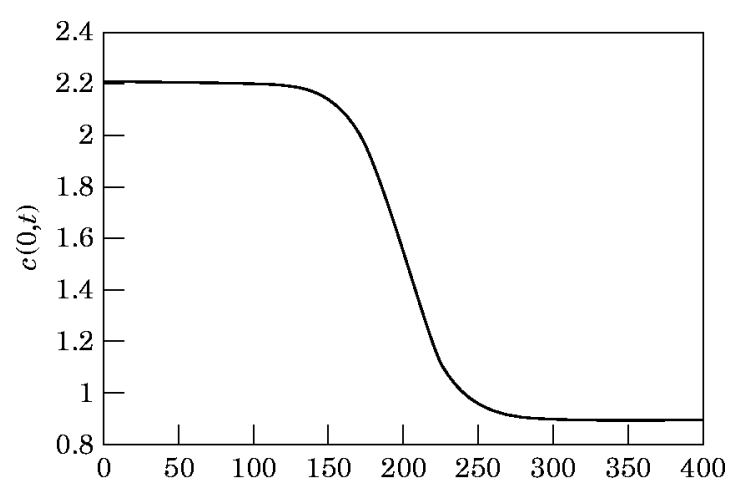

FIG. 6. Representative continuous function, used in simulations, for source of the inhibitor at the posterior edge of the jaw.

$$
c(0, t)=b \tanh \left[\frac{t-f}{g}\right]+h
$$

with

$$
b=-0.65, f=200, g=34, h=1.5 \text {. }
$$

and $c$ were given by the previous simulation except at time $t=0$, when $c(x, 0)=c_{0}(x)$ and $u$ and $v$ were at homogeneous steady states, with an added small random spatial perturbation. All simulations were run with the boundary conditions given in (9). Parameters were chosen so that the first unstable mode had the shape of a single peak in $u$ and $v$. We assume (reasonably from the biology) that the posterior end source, $c(0, t)=c_{0}(t)$, decays with time (Fig. 6). The diminishing role of the initial gradient in $c$, in favor of later placode-induced gradients, is in

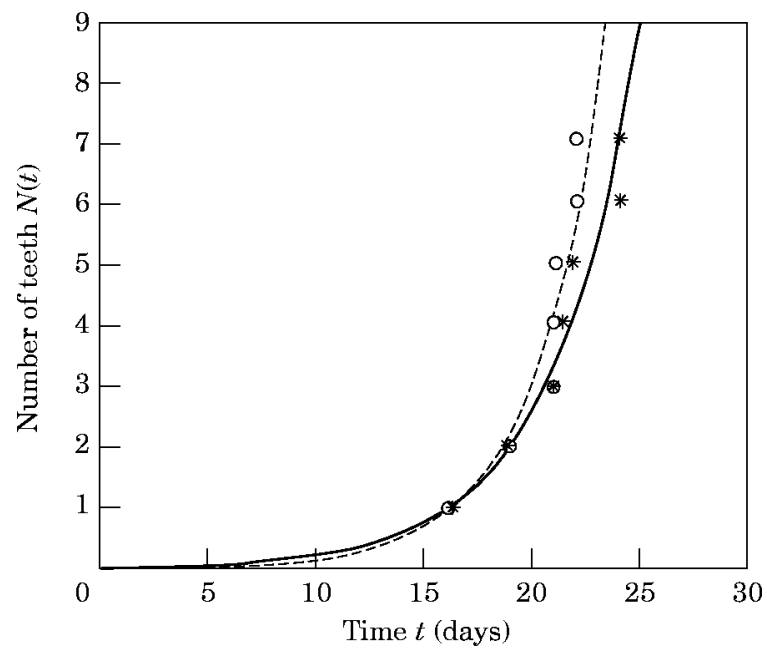

FIG. 7. Numerical and experimental data (Westergaard \& Ferguson, 1986). Number of teeth $N(t)$ vs. time $t$ (days) for the first seven teeth primordia in the lower half jaw of Alligator mississippiensis.

* denotes numerical data with solid line, $N(t)=N_{1} \exp \left(r_{1} t\right)$ 0 denotes experimental data with dashed line, $N(t)=N_{2} \exp \left(r_{2} t\right)$ $\left(N_{1}=0.018, N_{2}=0.0066, r_{1}=0.25 /\right.$ day, $r_{2}=0.31 /$ day $)$ Time $t$ (days) was scaled to time $T$ (simulation) using; $T=k t+b$, with $k=23.18 /$ day and $b=-225.8$. line with the hypothesis that early teeth primordia are crucial in the positioning of subsequent primordia. A comparison of the numerical simulation data, which show both position and order of appearance, vs. the experimental data (Westergaard \& Ferguson, 1986) of the first seven teeth is shown in Fig. 7.

Our model assumption to partition an interval into subdomains separated by a newly formed tooth, may have been important to the numerical predictions of the proper spatial and temporal order of the teeth primordia. We investigated this by removing the internal no-flux boundary condition (barrier to diffusion) at each new tooth site. We constructed an algorithm, based on the Crank-Nicolson finite difference iterative scheme, which allowed the continuous simulation of the teeth primordia
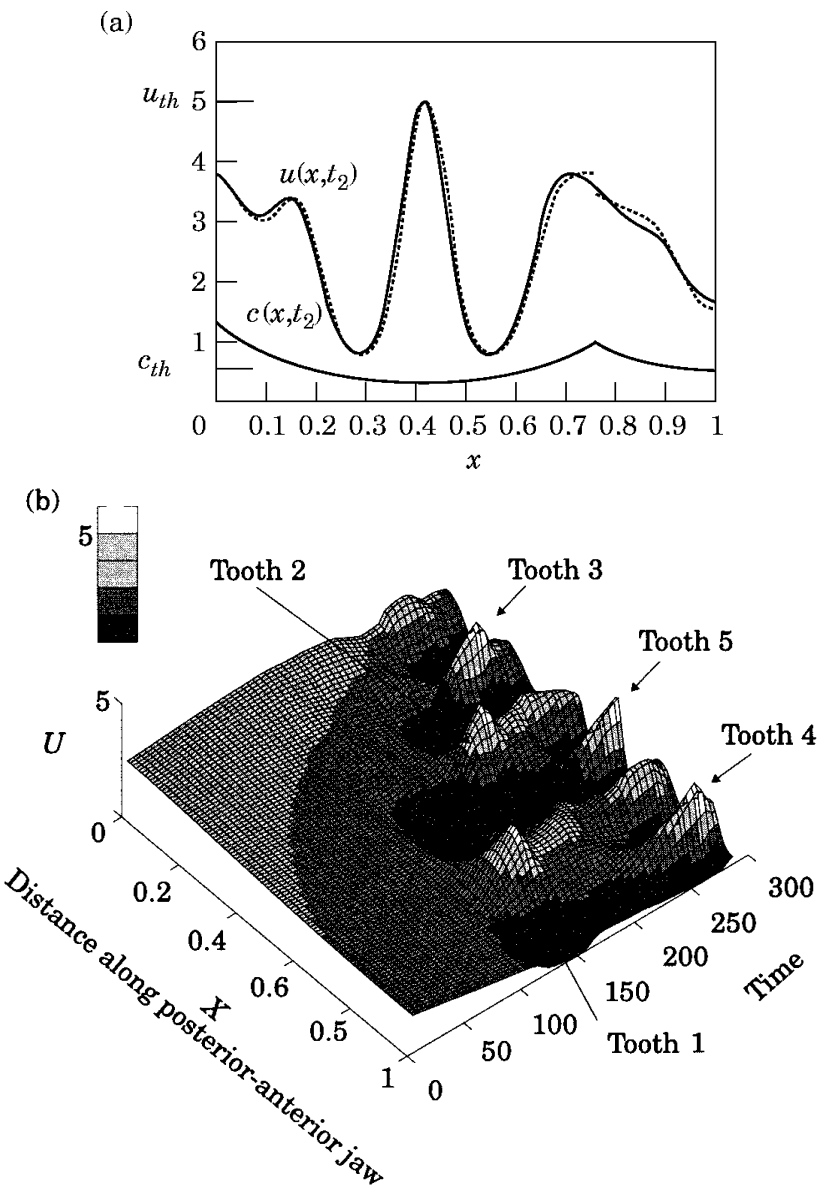

FIG. 8. (a) The initiation of the second tooth primordium. The solid line is the $u\left(x, t_{2}\right)$-substrate solution, calculated without the internal boundary conditions on $u$ and $v$. The dotted line is $u\left(x, t_{2}\right)$-substrate from Fig. 5. The numerical simulations use a finite-difference scheme based on the Crank-Nicolson method. The model parameters are the same as in Fig. 4. (b) Evolution of the $u(x, t)$-substrate conc. (vertically enhanced) during simulation on the jaw domain $0 \leqslant x \leqslant 1$. The internal no-flux boundary conditions on $u$ and $v$ are removed. The numerical scheme and model parameters are the same as in (a). 
sequence. We numerically solved the model system (6-8) with the boundary conditions given by (9a-e); the only boundary conditions on $u$ and $v$ were the no-flux conditions at the domain boundaries. Simulations have shown that the correct spatial and temporal sequence of the teeth primordia still develops without the original barriers (Fig. 8). These barriers, therefore, have very little, if any, effect on the development of the spatial patterning of the teeth primordia.

\section{Discussion}

We have developed a model mechanism based on biological facts, which can reproduce the spatiotemporal pattern of the first seven teeth primordia in the lower half jaw in A. mississippiensis. The pattern forms as a result of the intimate interaction of the pattern generator and jaw growth in a dynamic process. From experimental data (Westergaard \& Ferguson, 1986), the observed exponential jaw growth was incorporated directly into the model system. We have thus demonstrated that a reactiondiffusion type mechanism, when combined with the physical growth of the jaw, can reproduce the observed spatial patterning of Alligator teeth primordia.

The incorporation of exponential jaw growth directly into the model equations has offered a new mathematical challenge to our understanding of the properties of the pattern formation mechanism.

This work was in part supported by grants DMS-9106848 (PMK, JS, JDM, SRL), DMS-9500766 (JDM, SRL), and DMS-9306108 (SRL), from the U.S. National Science Foundation.

\section{REFERENCES}

Auchmuty, J. F. G. \& Nicolis, G. (1975). Bifurcation analysis of nonlinear reaction-diffusion equation-I. Evolution equations and the steady-state solutions. Bull. math. Biol. 37, 323-365.

Benson, D. L., Sherratt, J. A. \& Maini, P. K. (1993). Diffusion driven instability in an inhomogeneous domain. Bull. math. Biol. 55, 365-384.

Cantrell, R. S. \& Cosner, C. (1991). The effects of spatial heterogeneity in population dynamics. J. math. Biol. 29, 315-338.

Edmund, A. G. (1960). Evolution of dental patterns in the lower vertebrates. In: Evolution: Its Science and Doctrine. (Cameron, T. W. M., ed.) R. Soc. Can. Studia Varia. Ser. 4, pp. 45-62.

Ferguson, M. W. J. (1981). The value of the American alligator (Alligator mississippiensis) as a model for research in craniofacial development. J. craniofac. Genet. Devl. Biol. 1, 123-144.

Gierer, A. \& MEINHARDT, H. (1972). A theory of biological pattern formation. Kybernetik 12(1), 30-39.

Hill, R. E., Jones, F. J., Ree, A. R., Sime, C. M., Justice, M. J., Copeland, N. J., ET AL. (1989). A new family of mouse homeobox containing genes: molecular structure, chromosomal location and developmental expression. Genes Dev. 3, 26-37.
Jowett, A. K., Vainio, S., Ferguson, M. W. J., Sharpe, P. T. \& THESLEFF, I. (1993). Epithelial-mesenchymal interactions are required for msx 1 and msx 2 gene expression in the developing murine molar tooth. Development 117, 461-470.

Kollar, E. J. \& Mina, M. (1991). Role of the early epithelium in the patterning of the teeth and Meckel's cartilage. J. Craniofac. Genet. Dev. Biol. 11, 223-228.

Kronmiller, J. E., Upholt, W. B., Kollar, E. J. (1991). EGF Antisense oligodeoxynucleotides block murine odontogenesis in vitro. Dev. Biol. 147, 485-488.

Lumsden, A. G. S. (1988). Spatial organization of the epithelium and the role of neural crest cells in the initiation of the mammalian tooth germ. Development 103, (suppl.), 155-169.

MacKenzie, A., Leeming, G., Jowett, A. K., Ferguson, M. W. J. \& SHARPE, P. T. (1991). The homeobox gene Hox-7.1 has specific regional and temporal expression patterns during early murine craniofacial embryogenesis, especially tooth development in vivo and in vitro. Development 111, 269-285.

MacKenzie, A., Ferguson, M. W. J. \& Sharpe, P. T. (1992). Expression patterns of the homeobox gene, Hox-8, in the mouse embryo suggest a role in specifying tooth initiation and shape. Development 115, 403-420.

Mimura, M. \& Nishiura, Y. (1979). Spatial patterns for an interaction-diffusion equation in morphogenesis. J. math. Biol. 7, 243-263.

Mina, M. \& Kollar, E. J. (1987). The induction of odontogenesis in non-dental mesenchyme combined with early murine mandibular arch epithelium. Archs. oral Biol. 32(2), 123-127.

Monaghan, A. P., Hill, R. E., Bhattacharya, S. S., Graham, E. \& Davidson, D. R. (1991). Msh-like homeobox genes define domains in the developing vertebrate eye. Development 112, 1053-1061.

Murray, J. D. (1989). Mathematical Biology. 3rd Edn (1993) Heidelberg, Springer-Verlag.

OsboRn, J. W. (1971). The ontogeny of tooth succession in Lacerta vivipara Jacquin (1787). Proc. R. Soc. Lond. B179, 261-289.

Osborn, J. W. (1978). Morphogenetic gradients: fields versus clones. In: Development, function and evolution of teeth (Butler, P. M., Joysey, K. A., eds) pp. 171-201. New York: Academic Press.

Osborn, J. W. (1993). A Model Simulating Tooth Morphogenesis without Morphogens. J. theor. Biol. 165, 429-445.

Partanen, A. M. \& TheslefF, I. (1989). Growth factors and tooth development. Int. J. Dev. Biol. 33, 165-172.

Robert, B., Sassoon, D., JaQu, B., Gehring, W. \& Buckingham, M. (1989). Hox-7, a mouse homeobox gene with a novel pattern of expression during embryogenesis. Embo. J. 8, 91-100.

RösE, C. (1894). Ueber die Zahnentwicklung der Crocodile. Morph Arbeit. 3, 195-228.

SchnakenberG, J. (1979). Simple chemical reaction systems with limit cycle behaviour. J. theor. Biol. 81, 389-400.

Sneyd, J., Atri, A., Ferguson, M. W. J., Lewis, M. A., Seward, W. \& Murray, J. D. (1993). A model for the spatial patterning of teeth primordia in the Alligator: initiation of the dental determinant. J. theor. Biol. 165, 633-658.

Steinbock, O., Ketlunen, P., Showalter, K. (1995). Anistropy and spiral organizing centers in patterned excitable media. Science 269 (5232): 1857-62.

Turing, A. M. (1952). The chemical basis of morphogenesis. Phil. Trans. R. Soc. Lond. B237, 37-72.

Vainio, S., Karavanova, I., Jowett, A. K. \& TheslefF, I. (1993). Identification of BMP-4 as a signal mediating secondary induction between epithelial and mesenchymal tissues during early tooth development. Cell 75, 45-58.

Westergatrd, B. \& Ferguson, M. W. J. (1986). Development of the dentition in Alligator mississippiensis. Early embryonic development in the lower jaw. J. Zool. Lond. 210, 575-597.

Westergaard, B. \& Ferguson, M. W. J. (1987). Development of the dentition in Alligator mississippiensis. Later development in the lower jaws of embryos, hatchlings and young juveniles. J. Zool. Lond. 212, 191-222. 
Westergatrd, B. \& Ferguson, M. W. J. (1990). Development of the dentition in Alligator mississippiensis. Upper jaw dental and craniofacial development in embryos, hatchlings and young juveniles. Am. J. Anatomy 187, 393-421.

Woerdeman, M. W. (1919). Beitrage zur Entwicklungsgeschichte von Zahnen und Gebiss der Reptilien. Beitrag I. Die Anlage und Entwicklung des embryonalen Gebisses als Ganzes und seine Beziehung zur Zahnleiste. Arch. mikrosk. Anat. 92, 104-192.

WOLPERT, L. (1969). Positional information and the spatial pattern of cellular differentiation. J. theor. Biol. 25, 1-47.

\section{APPENDIX}

\section{Reaction-diffusion Systems on a Growing Domain}

Consider a jaw segment of length $L(t)$, which grows at a constant strain rate, $r$, so that

$$
\begin{gathered}
\frac{\partial L}{\partial t}=r L \\
L(t)=L_{0} e^{r t} .
\end{gathered}
$$

Consider a simple reaction-diffusion equation on the domain $0 \leqslant \xi \leqslant L$, growing at a constant strain rate, $r$, and which in the absence of growth is given by

$$
\frac{\partial c}{\partial t}=D \frac{\partial^{2} c}{\partial \xi^{2}}+f(c)
$$

where $D$ is the diffusion coefficient. Now let $L$ grow at a constant strain rate. Let $s$ represent the mass of reactant in length $L$, so that $c=s / L$. In the small time interval $(t, t+\Delta t)$, the length increases from $L$ to $L+\Delta L$ and the concentration changes from $c=s / L$, to $(s+\Delta s) /(L+\Delta L)$,

$$
\begin{aligned}
\Delta c & =\left[\frac{(s+\Delta s)}{(L+\Delta L)}\right]-\frac{s}{L} \\
& \approx\left[\frac{(s-L f(c) \Delta t)}{(L+r L \Delta t)}\right]-\frac{s}{L} \\
& \approx f(c) \Delta t-r c \Delta t
\end{aligned}
$$

which implies that

$$
\lim _{\Delta t \rightarrow 0}[\Delta c / \Delta t]=f(c)-r c .
$$

Equation (A.3) on the growing domain becomes,

$$
\frac{\partial c}{\partial t}=D \frac{\partial^{2} c}{\partial \xi^{2}}+f(c)-r c .
$$

Setting $x=\xi e^{-r t}$, where $x$ is the fixed domain variable, (A.5) becomes,

$$
\frac{\partial c}{\partial t}=D e^{-2 r t} \frac{\partial^{2} c}{\partial x^{2}}+f(c)-r c
$$

which represents a reaction-diffusion equation on a domain growing at a constant strain rate, $r$, transformed to a scaled domain $0 \leqslant x \leqslant L$ of constant length. 